\title{
One-pot, multicomponent synthesis of symmetrical Hantzsch 1,4-dihydropyridine derivatives using glycerol as clean and green solvent
}

\author{
Harvinder Singh Sohal a,*, Arun Goyal a, Rajeev Sharma ${ }^{\mathrm{b}}$ and Rajshree Khare a \\ a Department of Chemistry, Maharishi Markandeshwar University, Mullana-133 207, Haryana, India \\ b Department of Chemistry, Multani Mal Modi College, Patiala-147 001, Punjab, India \\ ${ }^{*}$ Corresponding author at: Department of Chemistry, Maharishi Markandeshwar University, Mullana-133 207, Haryana, India. \\ Tel.: +91.988.8857705. Fax: +91.1731.274375. E-mail address: luckysohal.singh@gmail.com (H. S. Sohal).
}

\section{ARTICLE INFORMATION}

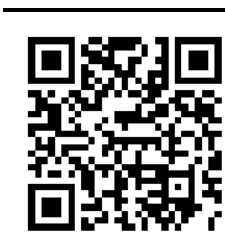

DOI: $10.5155 /$ eurjchem.5.1.171-175.943

Received: 05 October 2013

Received in revised form: 21 November 2013

Accepted: 27 November 2013

Online: 31 March 2014

\section{KEYWORDS}

\section{One-pot}

Glycerol

Aldehydes

Catalyst free

1,4-Dihydropyridine

Hantzsch Condensation

\section{Introduction}

The chemistry of 1,4-dihydropyridines (1,4-DHP's) found birth in 1882 with Hantzsch condensation [1]. After Hantzsch, multifarious new methods have been nurtured for the synthesis of original molecule. 1,4-DHP's attracted more attention, thanks to its presence in the coenzyme, diphospho pyridine nucleotide (DPNH) [2] and identification as bio-active material. In the present scenario many representatives have been commercialised such as nifedipine [3], felodipine [4], nicardipine [5], amlodipine [6] and even more have made their presence felt in the market [7] in the treatment of angina and hypertension. The activity profiles of 1,4-DHP's were further expanded as they were detected to possess anti-tumor [8], antiinflammatory [9], anticonvulsant activity [10], antitubercular activity $[11,12]$ cerebral antischemic activity in the treatment of Alzheimer's disease, PAF-acether antagonists [13]. Invention and execution of various new methodologies have engendered for the synthesis of symmetrical 1,4-DHP's. Off late many supported catalysts have been brought into use such as silica supported 12-tungstophosphoric acid [14]. Organo catalyst [15], [TBA $]_{2}\left[\mathrm{~W}_{6} \mathrm{O}_{19}\right]$ [16], Y(OTf) 3 [17] and use of nanoparticles also provided swiftness and higher degree of efficiency to the reaction such as Silicotungstic acid dispersed in the micropores if Cr-pillared clay [18], MgO [19] and cobalt [20] nanoparticles with the use of many instruments like microwave $[18,19,21$ $24]$, sonicator [25] turn out to exemplify. The above mentioned protocols have advantages over one another as they improve the Hantzsch condensation in terms of reaction time and yield [24]. However, the use of expensive catalysts and solvents does not allow the process to stay within the peripheries of a limited budget. In addition to this considering environmental and time perspective the process of combining solvent and catalysts cannot be believed to be undoubtly beneficial, the recovery of catalysts requiring a lot of solvent, time and purification based upon special methods could be termed as potent reasons. In the recent past reactions mediated with glycerol astonishing attention as glycerol is a solvent which is easily available and costs virtually nothing. In addition to this it does not distort the environmental processes. Not a long time ago, it was found that glycerol has been used for Heck and Suzuki coupling [26-28], Michael addition [29], Fridel-Crafts type addition, epoxide ring opening [30], synthesis of xanthenes [31] and very recently for the production of benzodiazepines and octahydroacridines $[32,33]$. Understanding the magnanimity of both 1,4-DHP's and glycerol, a new clean and green protocol has been discussed. In this effort, we synthesize 1,4-DHP's using glycerol as green solvent without amalgamatingany catalyst (Scheme 1). The present protocol is found to be much efficient over other procedure. 
Table 1. Effect of temperature on the synthesis of compounds $\mathbf{4 a}$

\begin{tabular}{lllll}
\hline S. No. & Compound & Temperature $\left.\mathbf{~}^{\circ} \mathbf{C}\right)$ & Time (minutes) & Yield a $(\%)$ \\
\hline 1 & $4 \mathrm{a}$ & 70 & 110 & 78 \\
2 & $4 \mathrm{a}$ & 80 & 85 & 83 \\
3 & $4 \mathrm{a}$ & 90 & 75 & 94 \\
4 & $4 \mathrm{a}$ & 100 & 74 & 94 \\
5 & $4 \mathrm{a}$ & 110 & 74 & 93 \\
\hline
\end{tabular}

a Yield refer to combined amounts of different crops.<smiles>[R]OC(=O)CC(C)=O</smiles>

1<smiles>[R]C=O</smiles>

3

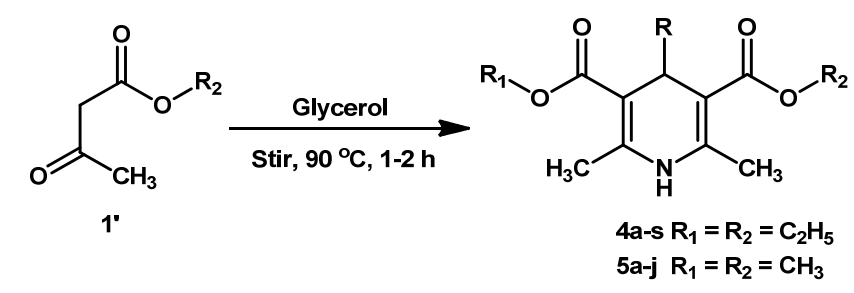

Scheme 1

\section{Experimental}

\subsection{Instrumentation}

Materials were obtained from commercial suppliers and were used without further purifications. Melting points were recorded in open end capillaries and are uncorrected. ${ }^{1} \mathrm{H}$ NMR spectra were recorded in DMSO- $d_{6}$ on a Bruker Avance II 400 $\mathrm{MHz}$ spectrometer; chemical shifts (delta) are reported in ppm relative to TMS as internal standard. The mass spectrum and IR spectra were recorded at LC-MS Spectrometer Model Q-ToF Micro Waters and Perkin-Elmer Spectrum II infra-red spectrophotometer, respectively. Elemental analyses $(\mathrm{C}, \mathrm{H}$, and N) were performed using a Thermo Scientific elemental analyser.

\subsection{Synthesis}

\subsubsection{Synthesis of diethyl 2,6-dimethyl-1,4-dihydropyridine- 3,5-dicarboxylate}

In a conical flask benzaldehyde $(0.01 \mathrm{~mol})$, ethyl acetoacetate $(0.02 \mathrm{~mol})$ and ammonium acetate $(0.02 \mathrm{~mol})$ were taken in a pre-heated glycerol $(10 \mathrm{~mL})$ and stirred at 90 ${ }^{\circ} \mathrm{C}$ for the stipulated time Table 1 . After the completion of reaction (vide TLC), reaction mixture was cooled to room temperature and added $50 \mathrm{~mL}$ ice-cold water, solid separated out. Filtered and dried, recrystallized from ethanol to afford compound 4a, 94\% yield, m.p.: $159-160{ }^{\circ} \mathrm{C}$ (Entry 1, Table 2). Similarly, other aldehydes $\mathbf{2 b}-\mathbf{k}$ were reacted with ethyl/methyl acetoacetate and ammonium acetate to afford various 1,4-dihydropyridines derivatives $\mathbf{4 b - q}$ and $\mathbf{5 a - k}$ (Table 2). Data obtained using advanced spectral techniques for some selected compounds have been summarized.

Diethyl 2,6-dimethyl-4-phenyl-1,4-dihydropyridine-3,5dicarboxylate (4a): Yield: 94\%. M.p.: 159-160 ${ }^{\circ} \mathrm{C}$. FT-IR (KBr, $v$, $\left.\mathrm{cm}^{-1}\right): 3340 v\left(\mathrm{~N}-\mathrm{H}\right.$ Str.), $1695 v\left(\mathrm{C}=0\right.$ Str.). ${ }^{1} \mathrm{H}$ NMR $(400 \mathrm{MHz}$, DMSO- $\left.d_{6}, \delta, \mathrm{ppm}\right): 1.21\left(\mathrm{t}, 6 \mathrm{H}, 2 \times \mathrm{CH}_{3}\right), 2.36\left(\mathrm{~s}, 6 \mathrm{H}, 2 \times \mathrm{CH}_{3}\right)$, 4.11 (q, 4H, $2 \times \mathrm{CH}_{2} \mathrm{CH}_{3}$ ), 4.98 (s, 1H, C-H), 6.01 (br s, 1H, N-H), 7.16-7.33 (m, 5H, Ar-H). MS (EI, $m / z,(\%)): 330\left(\mathrm{M}^{+}, 24\right)$. Anal. calcd. for $\mathrm{C}_{19} \mathrm{H}_{23} \mathrm{NO}_{4}$ : C, 69.28; H, 7.04; N, 4.25. Found: C, 69.25; H, 7.00; N, 4.24\%.

Diethyl 2,6-dimethyl-4-(4-methoxyphenyl)-1,4-dihydro pyridine-3,5-dicarboxylate (4c): Yield: $93 \%$. M. p.: 158-160 ${ }^{\circ} \mathrm{C}$. FT-IR (KBr, $\left.v, \mathrm{~cm}^{-1}\right): 3327 v$ (N-H Str.), $1699 v$ (C=0 Str.). ${ }^{1} \mathrm{H}$ NMR (400 MHz, DMSO- $\left.d_{6}, \delta, \mathrm{ppm}\right): 1.23\left(\mathrm{t}, 6 \mathrm{H}, 2 \times \mathrm{CH}_{3}\right), 2.32(\mathrm{~s}, 6 \mathrm{H}, 2$ $\mathrm{x} \mathrm{CH}$ ), 3.57 (s, 3H, OCH $), 4.15$ (q, 4H, $\left.2 \mathrm{x} \mathrm{CH}_{2} \mathrm{CH}_{3}\right), 4.95$ (s, $1 \mathrm{H}$, C-H), 6.11 (br s, 1H, N-H), 7.01-7.22 (m, 4H, Ar-H). MS (EI, $m / z$,
(\%)): $360\left(\mathrm{M}^{+}, 25\right)$. Anal. calcd. for $\mathrm{C}_{20} \mathrm{H}_{25} \mathrm{NO}_{5}$ : $\mathrm{C}, 66.83 ; \mathrm{H}, 7.01$; N, 3.90. Found: C, 66.81; H, 6.99; N, 3.89\%.

Diethyl 2,6-dimethyl-4-(3,4-dimethoxyphenyl)-1,4-dihydro pyridine-3,5-dicarboxylate (4d): Yield: $95 \%$. M.p.: $148-150{ }^{\circ} \mathrm{C}$. FT-IR (KBr, v, cm $\left.{ }^{-1}\right): 3341 v$ (N-H Str.), $1689 v\left(\mathrm{C}=0\right.$ Str.). ${ }^{1} \mathrm{H}$ NMR (400 MHz, DMSO- $\left.d_{6}, \delta, p p m\right): 1.24\left(\mathrm{t}, 6 \mathrm{H}, 2 \times \mathrm{CH}_{3}\right), 2.31(\mathrm{~s}, 6 \mathrm{H}, 2$ $\left.x \mathrm{CH}_{3}\right), 3.79$ (s, $\left.3 \mathrm{H}, \mathrm{OCH}_{3}\right), 3.85\left(\mathrm{~s}, 3 \mathrm{H}, \mathrm{OCH}_{3}\right), 4.12(\mathrm{q}, 4 \mathrm{H}, 2 \mathrm{x}$ $\left.\mathrm{CH}_{2} \mathrm{CH}_{3}\right), 4.90(\mathrm{~s}, 1 \mathrm{H}, \mathrm{C}-\mathrm{H}), 5.80(\mathrm{br} \mathrm{s}, 1 \mathrm{H}, \mathrm{N}-\mathrm{H}), 6.73-6.89(\mathrm{~m}$, $3 \mathrm{H}, \mathrm{Ar}-\mathrm{H})$. MS (EI, $m / z,(\%)): 390\left(\mathrm{M}^{+}, 25\right)$. Anal. calcd. for $\mathrm{C}_{21} \mathrm{H}_{27} \mathrm{NO}_{6}$ : C, 64.77; H, 6.99; N, 3.60. Found: C, 64.75; H, 6.97; N, $3.58 \%$.

Diethyl 2,6-dimethyl-4-(3,4,5-trimethoxyphenyl)-1,4-dihydro pyridine-3,5-dicarboxylate (4e): Yield: 91\%. M.p.: 184-186 ${ }^{\circ} \mathrm{C}$. FT-IR (KBr, v, cm-1): $3356 v\left(\mathrm{~N}-\mathrm{H}\right.$ Str.), $1704 v\left(\mathrm{C}=0\right.$ Str.). ${ }^{1} \mathrm{H}$ NMR (400 MHz, DMSO- $\left.d_{6}, \delta, p p m\right): 1.21\left(\mathrm{t}, 6 \mathrm{H}, 2 \times \mathrm{CH}_{3}\right), 2.30$ (s, $\left.6 \mathrm{H}, 2 \times \mathrm{CH}_{3}\right), 3.78\left(\mathrm{~s}, 3 \mathrm{H}, \mathrm{OCH}_{3}\right), 3.82\left(\mathrm{~s}, 6 \mathrm{H}, 2 \times \mathrm{OCH}_{3}\right) 4.11(\mathrm{q}$ $4 \mathrm{H}, 2 \times \mathrm{CH}_{2} \mathrm{CH}_{3}$ ), 4.93 (s, 1H, C-H), 5.91 (br s, $\left.1 \mathrm{H}, \mathrm{N}-\mathrm{H}\right), 6.52$ (s, $2 \mathrm{H}, \mathrm{Ar}-\mathrm{H})$. MS (EI, $m / z,(\%)): 420\left(\mathrm{M}^{+}, 26\right)$. Anal. Calcd. for $\mathrm{C}_{22} \mathrm{H}_{29} \mathrm{NO}_{7}$ : C, 62.99; H, 6.97; N, 3.34. Found: C, 62.96; H, 6.94; N, $3.31 \%$.

Diethyl 2,6-dimethyl-4-(3-nitrophenyl)-1,4-dihydropyridine3,5-dicarboxylate (4g): Yield: 93\%. M.p.: 155-166 ${ }^{\circ} \mathrm{C}$. FT-IR (KBr, v, cm-1): $3343 v\left(\mathrm{~N}-\mathrm{H}\right.$ Str.), $1703 v\left(\mathrm{C}=0\right.$ Str.). ${ }^{1} \mathrm{H}$ NMR (400 MHz, DMSO- $\left.d_{6}, \delta, \mathrm{ppm}\right): 1.25\left(\mathrm{t}, 6 \mathrm{H}, 2 \times \mathrm{CH}_{3}\right), 2.36(\mathrm{~s}, 6 \mathrm{H}, 2 \times$ $\left.\mathrm{CH}_{3}\right), 4.11$ (q, $\left.4 \mathrm{H}, 2 \times \mathrm{CH}_{2} \mathrm{CH}_{3}\right), 5.01(\mathrm{~s}, 1 \mathrm{H}, \mathrm{C}-\mathrm{H}), 6.08(\mathrm{br} \mathrm{s}, 1 \mathrm{H}$, $\mathrm{N}-\mathrm{H})$, 7.10-7.57 (m, 4H, Ar-H). MS (EI, $m / z,(\%)): 375\left(\mathrm{M}^{+}, 26\right)$. Anal. calcd. for $\mathrm{C}_{19} \mathrm{H}_{22} \mathrm{~N}_{2} \mathrm{O}_{6}$ : C, 60.95; H, 5.92; N, 7.48. Found: C, 60.93; H, 5.90; N, 7.47\%.

Diethyl 2,6-dimethyl-4-(4-nitrophenyl)-1,4-dihydropyridine3,5-dicarboxylate (4h): Yield: 92\%. M.p.: $159-160{ }^{\circ} \mathrm{C}$. FT-IR $\left(\mathrm{KBr}, v, \mathrm{~cm}^{-1}\right): 3350 v\left(\mathrm{~N}-\mathrm{H}\right.$ Str.), $1699 v\left(\mathrm{C}=0\right.$ Str.). ${ }^{1} \mathrm{H}$ NMR (400 MHz, DMSO- $\left.d_{6}, \delta, p p m\right): 1.27\left(\mathrm{t}, 6 \mathrm{H}, 2 \mathrm{x} \mathrm{CH}_{3}\right), 2.33(\mathrm{~s}, 6 \mathrm{H}, 2 \mathrm{x}$ $\left.\mathrm{CH}_{3}\right), 4.13$ (q, 4H, $\left.2 \times \mathrm{CH}_{2} \mathrm{CH}_{3}\right), 5.09(\mathrm{~s}, 1 \mathrm{H}, \mathrm{C}-\mathrm{H}), 6.09(\mathrm{br} \mathrm{s}, 1 \mathrm{H}$, $\mathrm{N}-\mathrm{H}), 7.16-7.44(\mathrm{~m}, 4 \mathrm{H}, \mathrm{Ar}-\mathrm{H})$. MS (EI, $m / z,(\%)): 375\left(\mathrm{M}^{+}, 25\right)$. Anal. calcd. for $\mathrm{C}_{19} \mathrm{H}_{22} \mathrm{~N}_{2} \mathrm{O}_{6}$ : C, 60.95; H, 5.92; N, 7.48. Found: C, 60.92; H, 5.91; N, 7.44\%.

Diethyl 2,6-dimethyl-4-(3-chlorophenyl)-1,4-dihydropyridine3,5-dicarboxylate (4i): Yield: 90\%. M.p.: 138-139 ${ }^{\circ} \mathrm{C}$. FT-IR (KBr, $v, \mathrm{~cm}^{-1}$ ): $3339 v$ (N-H Str.), $1699 v$ (C=0 Str.). ${ }^{1} \mathrm{H}$ NMR ( $400 \mathrm{MHz}$, DMSO- $\left.d_{6}, \delta, \mathrm{ppm}\right): 1.19\left(\mathrm{t}, 6 \mathrm{H}, 2 \times \mathrm{CH}_{3}\right), 2.31\left(\mathrm{~s}, 6 \mathrm{H}, 2 \times \mathrm{CH}_{3}\right)$, 4.07 (q, $\left.4 \mathrm{H}, 2 \times \mathrm{CH}_{2} \mathrm{CH}_{3}\right), 5.08(\mathrm{~s}, 1 \mathrm{H}, \mathrm{C}-\mathrm{H}), 5.97$ (br s, $\left.1 \mathrm{H}, \mathrm{N}-\mathrm{H}\right)$, 7.29-7.58 (m, 4H, Ar-H). MS (EI, $m / z,(\%)): 364\left(\mathrm{M}^{+}, 23\right)$. Anal. calcd. for $\mathrm{C}_{19} \mathrm{H}_{22} \mathrm{ClNO}_{4}$ : C, 62.72; $\mathrm{H}, 6.09 ; \mathrm{N}, 3.85$. Found: C, 62.71; H, 6.05; N, 3.84\%.

Diethyl 2,6-dimethyl-4-(4-chlorophenyl)-1,4-dihydropyridine3,5-dicarboxylate (4j): Yield: 93\%. M.p.: $147{ }^{\circ} \mathrm{C}$. FT-IR (KBr, v, $\mathrm{cm}^{-1}$ ): $3332 v(\mathrm{~N}-\mathrm{H}$ Str.), $1693 v(\mathrm{C}=0$ Str.). 


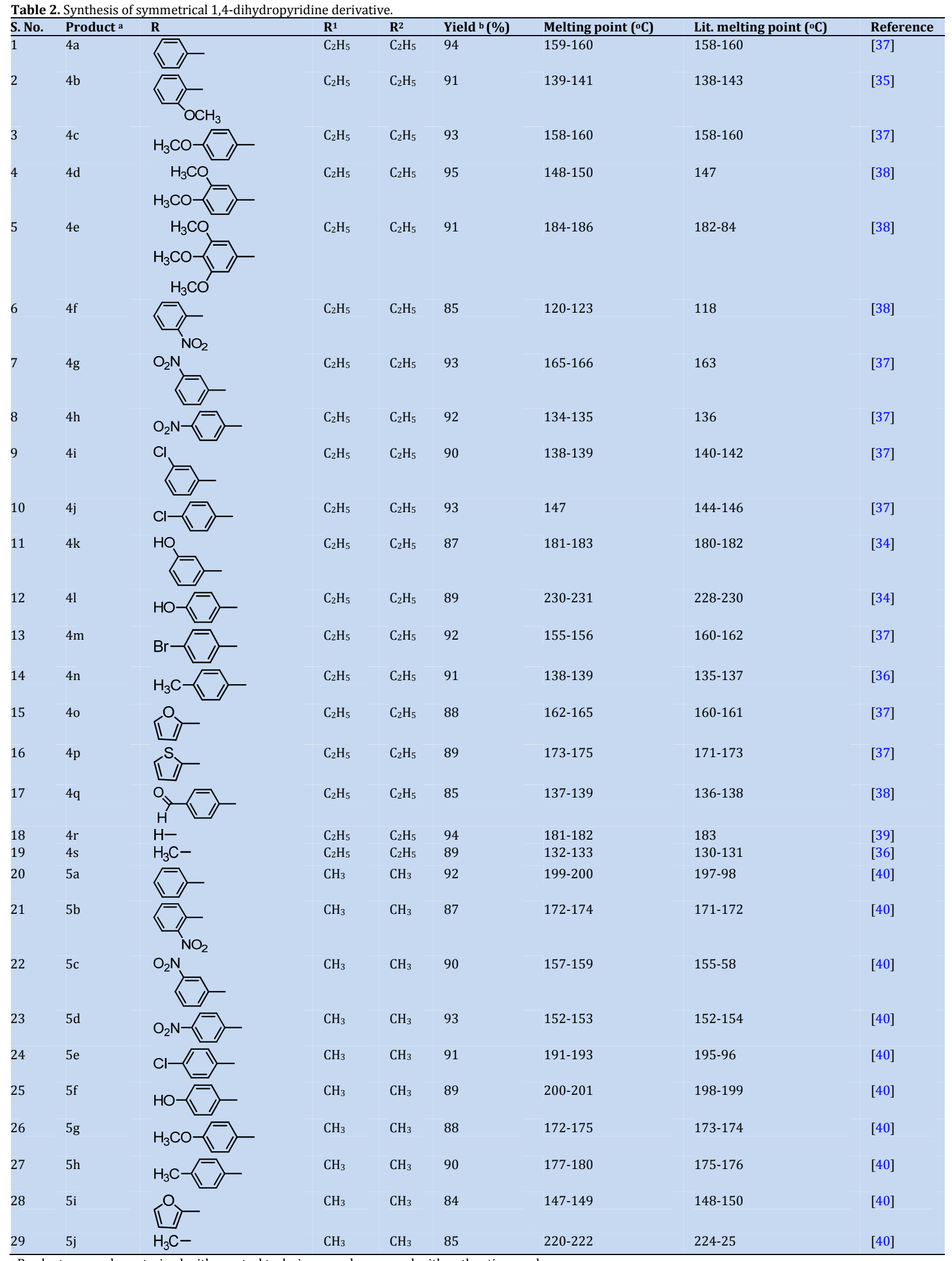

a Products were characterized with spectral techniques and compared with authentic samples.

b Yield refer to combined amounts of different crops. 
${ }^{1} \mathrm{H}$ NMR (400 MHz, DMSO- $\left.d_{6}, \delta, \mathrm{ppm}\right): 1.23\left(\mathrm{t}, 6 \mathrm{H}, 2 \times \mathrm{CH}_{3}\right)$, $2.36\left(\mathrm{~s}, 6 \mathrm{H}, 2 \times \mathrm{CH}_{3}\right), 4.13$ (q, $\left.4 \mathrm{H}, 2 \times \mathrm{CH}_{2} \mathrm{CH}_{3}\right), 5.11(\mathrm{~s}, 1 \mathrm{H}, \mathrm{C}-\mathrm{H})$, 5.99 (br s, 1H, N-H), 7.30-7.57 (m, 4H, Ar-H). MS (EI, $m / z,(\%))$ : $364\left(\mathrm{M}^{+}, 24\right)$. Anal. calcd. for $\mathrm{C}_{19} \mathrm{H}_{22} \mathrm{ClNO}_{4}$ : C, 62.72; H, 6.09; N, 3.85. Found: C, $62.71 ; \mathrm{H}, 6.08 ; \mathrm{N}, 3.82 \%$.

Diethyl 2,6-dimethyl-4-(3-hydroxyphenyl)-1,4-dihydro pyridine-3,5-dicarboxylate (4k): Yield: 87\%. M.p.: 181-183 ${ }^{\circ} \mathrm{C}$. FT-IR (KBr, v, cm-1): $3430 v(\mathrm{O}-\mathrm{H}$ Str.), $3331 v$ (N-H Str.), 1690 $v$ (C=0 Str.). ${ }^{1} \mathrm{H}$ NMR $\left(400 \mathrm{MHz}\right.$, DMSO- $\left.d_{6}, \delta, \mathrm{ppm}\right): 1.27(\mathrm{t}, 6 \mathrm{H}, 2$ x $\left.\mathrm{CH}_{3}\right), 2.21\left(\mathrm{~s}, 6 \mathrm{H}, 2 \times \mathrm{CH}_{3}\right), 4.10\left(\mathrm{q}, 4 \mathrm{H}, 2 \mathrm{x} \mathrm{CH}_{2} \mathrm{CH}_{3}\right), 4.99$ (s, 1H, C-H), 5.98 (br s, 1H, N-H), 7.10-7.34 (m, 4H, Ar-H), 9.79 (br, s, $1 \mathrm{H}, \mathrm{O}-\mathrm{H})$. MS (EI, $m / z,(\%)): 346\left(\mathrm{M}^{+}, 19\right)$. Anal. calcd. for $\mathrm{C}_{19} \mathrm{H}_{23} \mathrm{NO}_{5}$ : C, 66.07; H, 6.71; N, 4.06. Found: C, 66.06; H, 6.68; N, $4.03 \%$.

Diethyl 2,6-dimethyl-4-(4-hydroxyphenyl)-1,4-dihydro pyridine-3,5-dicarboxylate (41): Yield: 89\%. M.p.: 181-183 ${ }^{\circ} \mathrm{C}$. FT-IR (KBr, v, cm-1): 3447 v(O-H Str.), $3338 v(\mathrm{~N}-\mathrm{H}$ Str.), 1701 $v\left(\mathrm{C}=0\right.$ Str.). ${ }^{1} \mathrm{H}$ NMR $\left(400 \mathrm{MHz}, \mathrm{DMSO}-d_{6}, \delta, \mathrm{ppm}\right): 1.19(\mathrm{t}, 6 \mathrm{H}, 2$ x $\left.\mathrm{CH}_{3}\right), 2.30$ (s, 6H, $2 \times \mathrm{CH}_{3}$ ), 4.07 (q, $4 \mathrm{H}, 2 \mathrm{x} \mathrm{CH}_{2} \mathrm{CH}_{3}$ ), 5.01 (s, 1H, C-H), 6.08 (br s, 1H, N-H), 7.18-7.54 (m, 4H, Ar-H), 9.81 (br, s, $1 \mathrm{H}, \mathrm{O}-\mathrm{H})$. MS (EI, $m / z,(\%)): 346\left(\mathrm{M}^{+}, 21\right)$. Anal. calcd. for $\mathrm{C}_{19} \mathrm{H}_{23} \mathrm{NO}_{5}$ : C, 66.07; H, 6.71; N, 4.06. Found: C, 66.02; H, 6.71; N, 4.04\%.

Diethyl 2,6-dimethyl-4-(4-bromophenyl)-1,4-dihydro pyridine-3,5-dicarboxylate (4m): Yield: 92\%. M.p.: 155-156 ${ }^{\circ} \mathrm{C}$. FT-IR (KBr, v, cm-1): $3336 v\left(\mathrm{~N}-\mathrm{H}\right.$ Str.), $1703 v\left(\mathrm{C}=0\right.$ Str.). ${ }^{1} \mathrm{H}$ NMR (400 MHz, DMSO- $\left.d_{6}, \delta, \mathrm{ppm}\right): 1.21\left(\mathrm{t}, 6 \mathrm{H}, 2 \times \mathrm{CH}_{3}\right), 2.29$ (s, $\left.6 \mathrm{H}, 2 \times \mathrm{CH}_{3}\right), 4.09$ (q, 4H, $\left.2 \times \mathrm{CH}_{2} \mathrm{CH}_{3}\right), 5.03(\mathrm{~s}, 1 \mathrm{H}, \mathrm{C}-\mathrm{H}), 6.07(\mathrm{br}$ s, 1H, N-H), 7.13-7.51 (m, 4H, Ar-H). MS (EI, $m / z,(\%)): 409\left(\mathrm{M}^{+}\right.$, 25). Anal. calcd. for $\mathrm{C}_{19} \mathrm{H}_{22} \mathrm{BrNO}_{4}$ : C, 55.89; $\mathrm{H}, 5.43$; N, 3.43 . Found: C, 55.88; $\mathrm{H}, 5.41 ; \mathrm{N}, 3.39 \%$.

Diethyl 2,6-dimethyl-4-(4-methylphenyl)-1,4-dihydro pyridine-3,5-dicarboxylate (4n): Yield: $91 \%$. M.p.: $138-139{ }^{\circ} \mathrm{C}$. FT-IR (KBr, v, cm-1): $3329 v\left(\mathrm{~N}-\mathrm{H}\right.$ Str.), $1695 v\left(\mathrm{C}=0\right.$ Str.). ${ }^{1} \mathrm{H}$ NMR (400 MHz, DMSO- $\left.d_{6}, \delta, \mathrm{ppm}\right): 1.20\left(\mathrm{t}, 6 \mathrm{H}, 2 \mathrm{x} \mathrm{CH}_{3}\right), 2.19$ (s, $\left.3 \mathrm{H}, \mathrm{CH}_{3}\right), 2.21\left(\mathrm{~s}, 6 \mathrm{H}, 2 \times \mathrm{CH}_{3}\right), 4.03\left(\mathrm{q}, 4 \mathrm{H}, 2 \mathrm{x} \mathrm{CH}_{2} \mathrm{CH}_{3}\right), 5.07$ (s, 1H, C-H), 5.98 (br s, 1H, N-H), 7.15-7.53 (m, 4H, Ar-H). MS (EI, $m / z,(\%)): 343\left(\mathrm{M}^{+}, 20\right)$. Anal. calcd. for $\mathrm{C}_{20} \mathrm{H}_{25} \mathrm{NO}_{4}$ : C, 69.95; $\mathrm{H}$, 7.34; N, 4.08. Found: C, 69.88; H, 7.31; N, 4.02\%.

Diethyl 2,6-dimethyl-4-(2-furyl)-1,4-dihydropyridine-3,5dicarboxylate (4o): Yield: 88\%. M.p.: $162-165^{\circ} \mathrm{C}$. FT-IR (KBr, $v$, $\left.\mathrm{cm}^{-1}\right)$ : $3335 \mathrm{v}\left(\mathrm{N}-\mathrm{H}\right.$ Str.), $1701 \mathrm{v}\left(\mathrm{C}=0\right.$ Str.). ${ }^{1} \mathrm{H}$ NMR $(400 \mathrm{MHz}$, DMSO- $\left.d_{6}, \delta, \mathrm{ppm}\right): 1.27\left(\mathrm{t}, 6 \mathrm{H}, 2 \times \mathrm{CH}_{3}\right), 2.31\left(\mathrm{~s}, 6 \mathrm{H}, 2 \times \mathrm{CH}_{3}\right)$, 4.17 (q, $\left.4 \mathrm{H}, 2 \mathrm{x} \mathrm{CH}_{2} \mathrm{CH}_{3}\right), 4.99(\mathrm{~s}, 1 \mathrm{H}, \mathrm{C}-\mathrm{H}), 6.07$ (br s, $\left.1 \mathrm{H}, \mathrm{N}-\mathrm{H}\right)$, 6.33-6.42 (m, 2H, Furyl-H), 7.17 (m, 1H, Furyl-H). MS (EI, $m / z$, (\%)): $320\left(\mathrm{M}^{+}, 16\right)$. Anal. calcd. for $\mathrm{C}_{17} \mathrm{H}_{21} \mathrm{NO}_{5}$ : $\mathrm{C}, 63.94 ; \mathrm{H}, 6.63$; $\mathrm{N}, 4.39$. Found: $\mathrm{C}, 63.92 ; \mathrm{H}, 6.62 ; \mathrm{N}, 4.37 \%$.

Diethyl 2,6-dimethyl-4-(2-theniyl)-1,4-dihydropyridine-3,5dicarboxylate (4p): Yield: 89\%. M.p.: $173-175{ }^{\circ} \mathrm{C}$. FT-IR (KBr, v, $\left.\mathrm{cm}^{-1}\right): 3345 v\left(\mathrm{~N}-\mathrm{H}\right.$ Str.), $1699 v\left(\mathrm{C}=0\right.$ Str.). ${ }^{1} \mathrm{H}$ NMR $(400 \mathrm{MHz}$, DMSO- $\left._{6}, \delta, \mathrm{ppm}\right): 1.17\left(\mathrm{t}, 6 \mathrm{H}, 2 \times \mathrm{CH}_{3}\right), 2.30\left(\mathrm{~s}, 6 \mathrm{H}, 2 \times \mathrm{CH}_{3}\right)$, 4.06 (q, $\left.4 \mathrm{H}, 2 \times \mathrm{CH}_{2} \mathrm{CH}_{3}\right), 5.01$ (s, $\left.1 \mathrm{H}, \mathrm{C}-\mathrm{H}\right), 6.06$ (br s, $\left.1 \mathrm{H}, \mathrm{N}-\mathrm{H}\right)$, 6.09-6.14 (m, 2H, Thienyl-H), 6.89 (m, 1H, Thienyl-H). MS (EI, $m / z,(\%)): 336\left(\mathrm{M}^{+}, 18\right)$. Anal. calcd. for $\mathrm{C}_{17} \mathrm{H}_{21} \mathrm{NO}_{4} \mathrm{~S}: \mathrm{C}, 60.87 ; \mathrm{H}$, 6.31; N, 4.18. Found: C, 60.85; H, 6.29; N, 4.17\%.

Diethyl 2,6-dimethyl-4-(4-formylphenyl)-1,4-dihydro pyridine-3,5-dicarboxylate (4q): Yield: $85 \%$. M.p.: $137-139{ }^{\circ} \mathrm{C}$. FT-IR (KBr, v, cm-1): $3349 v\left(\mathrm{~N}-\mathrm{H}\right.$ Str.), $1706 v\left(\mathrm{C}=\mathrm{O}\right.$ Str.). ${ }^{1} \mathrm{H}$ NMR (400 MHz, DMSO- $\left.d_{6}, \delta, \mathrm{ppm}\right): 1.19\left(\mathrm{t}, 6 \mathrm{H}, 2 \mathrm{x} \mathrm{CH}_{3}\right), 2.32(\mathrm{~s}$, $\left.6 \mathrm{H}, 2 \times \mathrm{CH}_{3}\right), 4.07\left(\mathrm{q}, 4 \mathrm{H}, 2 \times \mathrm{CH}_{2} \mathrm{CH}_{3}\right), 5.02(\mathrm{~s}, 1 \mathrm{H}, \mathrm{C}-\mathrm{H}), 5.96(\mathrm{br}$ s, 1H, N-H), 7.49-7.80 (m, 4H, Ar-H), 8.92 (s, 1H, CHO). MS (EI, $m / z,(\%)): 358\left(\mathrm{M}^{+}, 23\right)$. Anal. calcd. for $\mathrm{C}_{20} \mathrm{H}_{23} \mathrm{NO}_{5}$ : C, 67.21; $\mathrm{H}$, 6.49; N, 3.92. Found: C, 67.20; H, 6.47; N, 3.89\%.

Dimethyl 2,6-dimethyl-4-phenyl-1,4-dihydropyridine-3,5dicarboxylate (5a): Yield: 92\%. M.p.: 199-200 ${ }^{\circ} \mathrm{C}$. FT-IR (KBr, v, $\left.\mathrm{cm}^{-1}\right)$ : $3320 v\left(\mathrm{~N}-\mathrm{H}\right.$ Str.), $1690 v\left(\mathrm{C}=0\right.$ Str.). ${ }^{1} \mathrm{H}$ NMR (400 MHz, DMSO- $d_{6}, \delta$, ppm): 2.25 (s, 6H, $\left.2 \times \mathrm{CH}_{3}\right), 3.68$ (s, 6H, $2 \times \mathrm{CH}_{3}$ ), $4.99(\mathrm{~s}, 1 \mathrm{H}, \mathrm{C}-\mathrm{H}), 6.04$ (br s, 1H, N-H), 6.94-7.34 (m, 5H, Ar-H). MS (EI, $m / z,(\%)): 302\left(\mathrm{M}^{+}, 25\right)$. Anal. calcd. for $\mathrm{C}_{17} \mathrm{H}_{19} \mathrm{NO}_{4}$ : C, 67.76; H, 6.36; N, 4.65. Found: C, 67.72; H, 6.34; N, 4.65\%.
Dimethyl 2,6-dimethyl-4-(4-nitrophenyl)-1,4-dihydro pyridine-3,5-dicarboxylate (5d): Yield: 93\%. M.p.: 152-153 ${ }^{\circ} \mathrm{C}$. FT-IR (KBr, v, cm $\left.{ }^{-1}\right): 3342 v\left(\mathrm{~N}-\mathrm{H}\right.$ Str.), $1707 v\left(\mathrm{C}=0\right.$ Str.). ${ }^{1} \mathrm{H}$ NMR (400 MHz, DMSO- $\left.d_{6}, \delta, \mathrm{ppm}\right): 2.28$ (s, $6 \mathrm{H}, 2 \mathrm{x} \mathrm{CH}_{3}$ ), 3.74 (s, $6 \mathrm{H}, 2 \times \mathrm{CH}_{3}$ ), 5.03 (s, 1H, C-H), 6.07 (br s, $\left.1 \mathrm{H}, \mathrm{N}-\mathrm{H}\right), 7.12-7.51$ (m, 4H, Ar-H). MS (EI, $m / z,(\%)): 347\left(\mathrm{M}^{+}, 24\right)$. Anal. calcd. for $\mathrm{C}_{17} \mathrm{H}_{18} \mathrm{~N}_{2} \mathrm{O}_{6}$ : C, 58.96; H, 5.24; N, 8.09. Found: $\mathrm{C}, 58.94 ; \mathrm{H}, 5.22$; $\mathrm{N}, 8.08 \%$.

Dimethyl 2,6-dimethyl-4-(4-chlorophenyl)-1,4-dihydro pyridine-3,5-dicarboxylate (5e): Yield: $91 \%$. M.p.: 191-193 ${ }^{\circ} \mathrm{C}$. FT-IR (KBr, v, cm $\left.{ }^{-1}\right): 3317 v\left(\mathrm{~N}-\mathrm{H}\right.$ Str.), $1700 v\left(\mathrm{C}=0\right.$ Str.). ${ }^{1} \mathrm{H}$ NMR (400 MHz, DMSO- $\left.d_{6}, \delta, \mathrm{ppm}\right): 2.26\left(\mathrm{~s}, 6 \mathrm{H}, 2 \times \mathrm{CH}_{3}\right), 3.70(\mathrm{~s}$, $\left.6 \mathrm{H}, 2 \mathrm{x} \mathrm{CH}_{3}\right), 5.02(\mathrm{~s}, 1 \mathrm{H}, \mathrm{C}-\mathrm{H}), 6.01$ (br s, $\left.1 \mathrm{H}, \mathrm{N}-\mathrm{H}\right), 7.01-7.37$ (m, 4H, Ar-H). MS (EI, $m / z,(\%)): 336\left(\mathrm{M}^{+}, 25\right)$. Anal. calcd. for $\mathrm{C}_{17} \mathrm{H}_{18} \mathrm{ClNO}_{4}$ : C, 60.81; H, 5.40; N, 4.17. Found: C, 60.79; H, 5.35; $\mathrm{N}, 4.15 \%$.

Dimethyl 2,6-dimethyl-4-(4-methoxyphenyl)-1,4-dihydro pyridine-3,5-dicarboxylate (5g): M.p.: 172-175 ${ }^{\circ} \mathrm{C}$. FT-IR (KBr, v, $\mathrm{cm}^{-1}$ ): $3295 \mathrm{v}$ (N-H Str.), $1691 \mathrm{v}\left(\mathrm{C}=0\right.$ Str.). ${ }^{1} \mathrm{H}$ NMR $(400 \mathrm{MHz}$, DMSO- $\left.d_{6}, \delta, \mathrm{ppm}\right): 2.23\left(\mathrm{~s}, 6 \mathrm{H}, 2 \mathrm{x} \mathrm{CH}_{3}\right), 3.68\left(\mathrm{~s}, 6 \mathrm{H}, 2 \times \mathrm{OCH}_{3}\right)$, $3.92\left(\mathrm{~s}, 3 \mathrm{H}, \mathrm{OCH}_{3}\right), 4.97(\mathrm{~s}, 1 \mathrm{H}, \mathrm{C}-\mathrm{H}), 5.94(\mathrm{br} \mathrm{s}, 1 \mathrm{H}, \mathrm{N}-\mathrm{H}), 6.89-$ 7.25 (m, 4H, Ar-H). MS (EI, $m / z,(\%)): 332\left(\mathrm{M}^{+}, 24\right)$. Anal. calcd. for $\mathrm{C}_{18} \mathrm{H}_{21} \mathrm{NO}_{5}$ : C, 65.24; $\mathrm{H}, 6.39$; $\mathrm{N}, 4.23$. Found: $\mathrm{C}, 65.22 ; \mathrm{H}$, 6.38; N, $4.20 \%$.

Dimethyl 2,6-dimethyl-4-(4-methylphenyl)-1,4-dihydro pyridine-3,5-dicarboxylate (5h): Yield: $90 \%$. M.p.: 177-180 ${ }^{\circ} \mathrm{C}$. FT-IR (KBr, v, cm-1): $3312 v\left(\mathrm{~N}-\mathrm{H}\right.$ Str.), $1689 v\left(\mathrm{C}=0\right.$ Str.). ${ }^{1} \mathrm{H}$ NMR (400 MHz, DMSO- $\left.d_{6}, \delta, \mathrm{ppm}\right): 2.18\left(\mathrm{~s}, 3 \mathrm{H}, \mathrm{CH}_{3}\right) 2.24(\mathrm{~s}, 6 \mathrm{H}$, $\left.2 \mathrm{x} \mathrm{CH}_{3}\right), 3.66\left(\mathrm{~s}, 6 \mathrm{H}, 2 \times \mathrm{CH}_{3}\right), 4.96(\mathrm{~s}, 1 \mathrm{H}, \mathrm{C}-\mathrm{H}), 5.92(\mathrm{br} \mathrm{s}, 1 \mathrm{H}$, $\mathrm{N}-\mathrm{H}), 6.90-7.31$ (m, 4H, Ar-H). MS (EI, $m / z,(\%)): 316\left(\mathrm{M}^{+}, 23\right)$. Anal. calcd. for $\mathrm{C}_{18} \mathrm{H}_{21} \mathrm{NO}_{4}$ : C, 68.55; $\mathrm{H}, 6.71 ; \mathrm{N}, 4.44$. Found: $\mathrm{C}$, 68.54; H, 6.69; N, 4.41\%.

Dimethyl 2,6-dimethyl-4-(2-furyl)-1,4-dihydropyridine-3,5dicarboxylate (5i): Yield: $84 \%$. M.p.: $147-149^{\circ} \mathrm{C}$. FT-IR (KBr, v, $\mathrm{cm}^{-1}$ ): $3317 v\left(\mathrm{~N}-\mathrm{H}\right.$ Str.), $1700 v\left(\mathrm{C}=0\right.$ Str.). ${ }^{1} \mathrm{H}$ NMR (400 MHz, DMSO- $\left.d_{6}, \delta, \mathrm{ppm}\right): 2.20\left(\mathrm{~s}, 6 \mathrm{H}, 2 \times \mathrm{CH}_{3}\right), 3.65\left(\mathrm{~s}, 6 \mathrm{H}, 2 \times \mathrm{CH}_{3}\right)$, 4.98 (s, 1H, C-H), 6.05 (br s, 1H, N-H), 6.33-6.45 (m, 2H, FurylH), 7.22 (m, 1H, Furyl-H). MS (EI, $m / z,(\%)): 292\left(\mathrm{M}^{+}, 18\right)$. Anal. calcd. for $\mathrm{C}_{15} \mathrm{H}_{17} \mathrm{NO}_{5}$ : C, 61.85; H, 5.88; N, 4.81. Found: C, 61.82; $\mathrm{H}, 5.87 ; \mathrm{N}, 4.78 \%$.

\section{Results and discussion}

Condensation of ethylacetoacetate (1), benzaldehyde (2a) and ammonium acetate (3) were carried out in glycerol at different temperatures $\left(70-110^{\circ} \mathrm{C}\right)$. It was observed that $90^{\circ} \mathrm{C}$ is the optimal temperature for the synthesis of 1,4dihydropyridines. Further rise in temperature had a negligible impact on rate and yield of the reaction (Table 1)

The structure of the compound $\mathbf{4 a}$ was confirmed with the use of spectral techniques. In IR spectrum absorption at 3340 $\mathrm{cm}^{-1}$ represents the $\mathrm{N}-\mathrm{H}$ stretching, a strong absorption for $\mathrm{C}=\mathrm{O}$ groups was observed at $1695 \mathrm{~cm}^{-1}$. In ${ }^{1} \mathrm{H}$ NMR spectra peaks for five aromatic protons are observed at $\delta 7.16-7.33 \mathrm{ppm}$, singlet at $\delta 4.98 \mathrm{ppm}$ for $-\mathrm{CH}$ proton, singlet at $\delta 6.01 \mathrm{ppm}$ for $-\mathrm{NH}$ proton and a singlet for two $-\mathrm{CH}_{3}$ groups observed at $\delta 2.36$ ppm, a triplet for two $-\mathrm{CH}_{3}$ groups observed at $\delta 1.21 \mathrm{ppm}$ and a quartet for two $-\mathrm{CH}_{2}$ groups was observed at $\delta 4.11 \mathrm{ppm}$. Spectral data of compound 4a fully supports the structure assigned to it. Similarly, other dialkyl-2,6-dimethyl-4-aryl-1,4dihydropyridine-3,5-dicarboxylate $\mathbf{4 b - p}$ and $\mathbf{5 a - k}$ have been synthesized by the condensation of ethyl/methyl acetoacetate (1), aldehyde (2) and ammonium acetate (3) in glycerol. The results are summarized in Table 2 .

In the proposed mechanism, for the synthesis of dihydropyridines follow the addition of compound $\mathbf{1}$ and $\mathbf{3}$ to give compound 6 by the removal of an acetic acid molecule and at the same time Knoevenagal condensation between compounds $\mathbf{1}$ and $\mathbf{2}$ to give compound 7, which upon Michael addition with compound $\mathbf{6}$ produce compound $\mathbf{8}$ them followed 

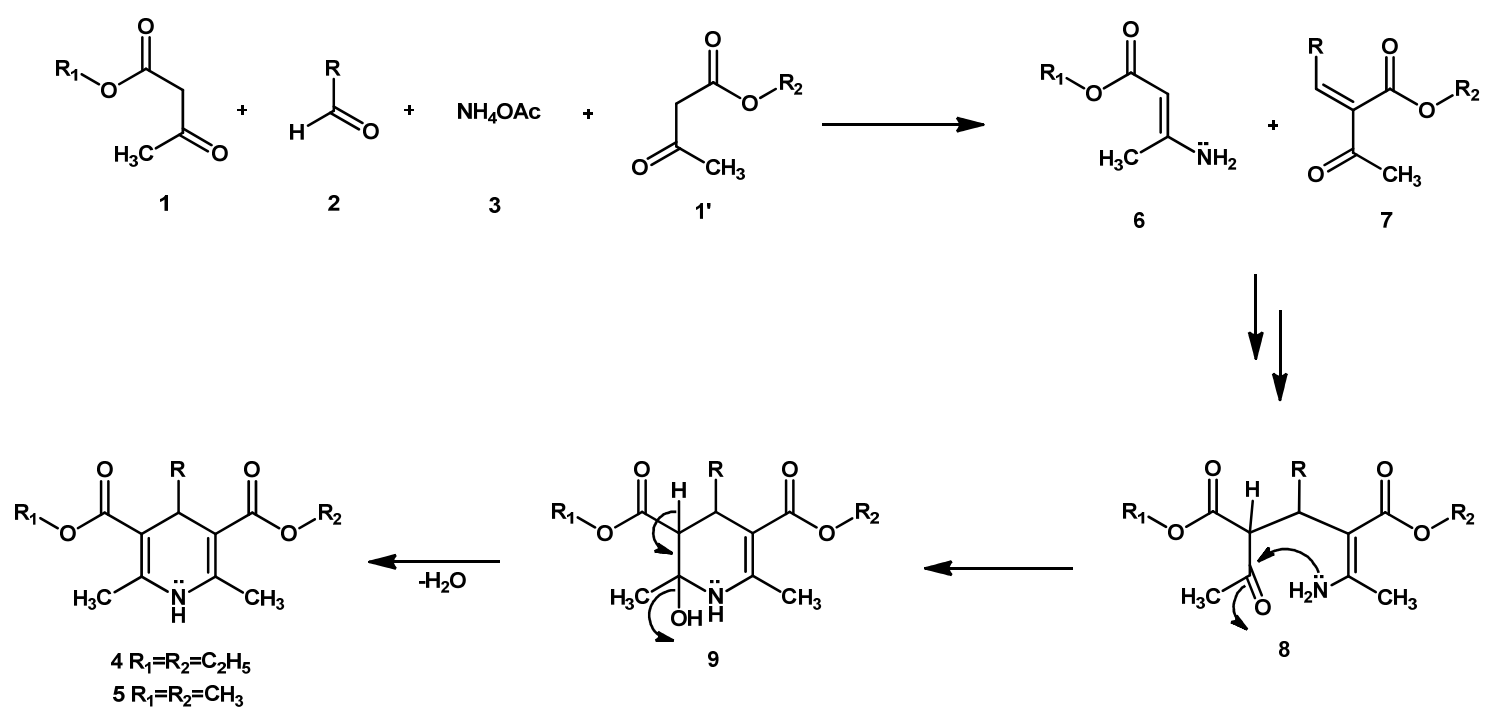

Scheme 2

by cyclization to produce 9 and rearrange to yield the 1,4-DHP molecule (4a-s and 5a-j) (Scheme 2).

Reactions proceeded smoothly with aldehydes carrying electron withdrawing as well as electron donating substituents (Table 2). This method endures various functionalities like nitro, ether, halogen etc. on the aldehydes. Efficacy of this method is fairly general and affords the resultant products in excellent yield (85-95\%) and products are obtained by simple work up.

\section{Conclusion}

The present procedure is an effective method for production of tetra substituted 1,4-dihydropyridine from readily available starting materials in a single step with inherent flexibility and diversity. This method was efficacious to reduce labor, cost, waste production and also devoid of harsh reaction conditions. The target compounds were obtained in an acceptable yield with simple recrystallization as a purification step.

\section{Acknowledgements}

The authors thank Maharishi Markandeshwar University, Mullana, Ambala, India for the financial support and Harvinder Singh Sohal and Arun Goyal also thank Mr. Vikas Pahwa for the liberal support.

\section{References}

[1]. Hantzsch, A. Jusfus Liebigs Ann. Chem. 1882, 215, 1-82.

[2]. Hutton, R. F.; Westheimer, F. H. Tetrahedron 1958, 3, 73-74.

[3]. Rahway, N. J. The Merck Index, 12th edition, Merck Research Laboratories, 1996.

[4]. Bostrom, S. L.; Ljung, B.; Mardh, S.; Forsen, S.; Thulin, E. Nature 1981, $292,777-778$

[5]. Iwanami, M.; Shibanuma, T.; Fujimoto, M.; Kawai, R.; Tamazawa, K.; Takenaka, T.; Takahashi, K.; Murakami, M. Chem. Pharm. Bull. 1979, 27, 1426-1440.

[6]. Arrowsmith, J. E.; Campbell, S. F.; Cross, P. E.; Stubbs, J. K.; Burges, R. A.; Gardiner, D. G.; Blackburn, K. J.J. Med. Chem. 1986, 29, 1696-1702.

[7]. Goldmann, S.; Stoltefuss, J. Angew. Chem., Int. Ed. Engl. 1991, 30, 15591578.

[8]. Boer, R.; Gekeler, V. Drugs Future 1995, 20, 499-509.

[9]. Briukhanov, V. M.; Zverev-laf Elkin, V. I. Exp. Clin. Pharmacol. 1994, 57, 47-49.

[10]. Tusell, J. M.; Serratosa, S. J. Brain Res. 1993, 622, 99-104.
[11]. Wachter, G. A.; Davis, M. C.; Martin, A. R.; Franzblau, S. G. J. Med. Chem. 1998, 41, 2436-2438.

[12]. Desai, B.; Sureja, D.; Naliapara, Y.; Shah, A.; Saxena, A. K. Bioorg. Med. Chem. 2001, 9, 1993-1998.

[13]. Sunkel, C. E.; de Casa-Juana, M. F.; Santos, L.; Gomez, M. M.; Villarroya, M.; Gonzalez-Morales, M. A.; Priego, J. G.; Ortega, M. P. J. Med. Chem. 1990, 33, 3205-3210.

[14]. Rafiee, E.; Eavani, S.; Rashidzadeh, S.; Joshaghani, M. Inorg. Chim. Acta 2009, 362, 3555-3562.

[15]. Kumar, A.; Maurya, R. A. Tetrahedron 2008, 64, 3477-3482.

[16]. Davoodnia, A.; Khashi, M.; Niloofar, T. H. Chinese J. Catal. 2013, 34, 1173-1178.

[17]. Ramchander, J.; Raju, G.; Rameshwar, N.; Reddy, T. S.; Reddy A. R. Spectrochimica Acta A 2012, 85, 210-216.

[18]. Kar, P.; Mishra, B. G. Chem. Eng. J. 2013, 223, 647-656,

[19]. Mirzaei, H.; Davoodnia, A. Chin. J. Catal. 2012, 33, 1502-1507.

[20]. Safari, J.; Banitaba, S. H.; Khalil, S. D. Chin. J. Catal. 2011, 32, 18501854.

[21]. Lee, Y. A.; Kim, S. C. J. Ind. Eng. Chem. 2011, 17, 401-403.

[22]. Chhillar, A. K.; Arya, P.; Mukherjee, C.; Kumar, P.; Yadav, Y.; Sharma, A K.; Yadav, V.; Gupta, J.; Dabur, R.; Jha, H. N.; Watterson, A. C.; Parmar, V. S.; Prasadb, A. K.; Sharma, G. L. Bioorg. Med. Chem. 2006, 14, 973-981.

[23]. Kuraitheerthakumaran, A.; Pazhamalai, S.; Gopalakrishnan, M. Chinese Chem. Lett. 2011, 22, 1199-1202.

[24]. Kotharkar, S. A.; Shinde, D. B. Ukrainica Bioorg. Acta 2006, 1, 3-5.

[25]. Wang, S. X.; Li, Z. Y.; Zhang, j. C.; Li, J. T. Ultrason. Sonochem. 2008, 15, 677-680.

[26]. Wolfson, A.; Litvak, G.; Dlugy, C.; Shotland, Y.; Tavor, D. Ind. Crops. Prod. 2009, 30, 78-81.

[27]. Wolfson, A.; Dlugy, C. Chem. Pap. 2007, 61, 228-232.

[28]. Wolfson, A.; Dlugy, C.; Shotland, Y. Environ. Chem. Lett. 2007, 5, 67-71.

[29]. Gu, Y.; Barrault, J.; Jerome, F. Adv. Synth. Catal. 2008, 350, 2007-2012.

[30]. Karam, A.; Villandier, N.; Delample, M.; Koerkamp, C. K.; Douliez, J. P. Granet, R.; Krausz, P.; Barrault, J.; Jerome, F. Chem. Eur. J. 2008, 14, 10196-10200.

[31]. He, F.; Li, P.; Gu, Y.; Li, G. Green Chem. 2009, 11, 1767-1773.

[32]. Radatz, C. S.; Silva, R. B.; Perin, G.; Lenardao, E. J.; Jacob, R. G.; Alves, D. Tetrahedron Lett. 2011, 52, 4132-4136.

[33]. Nascimento, J. E. R.; Barcellos, A. M.; Sachini, M.; Perin, G.; Lenardao, E. J.; Alves, D.; Jacob, R. G.; Missau, F. Tetrahedron Lett. 2011, 52, 2571 2574.

[34]. Tamaddon, F.; Razmi, Z:; Jafari, A. A. Tetrahedron Lett. 2010, 51, 11871189.

[35]. Love, B.; Goodman, M. M.; Snader, K. M.; Tedeschi, R.; Macko, E. Eur. J. Med. Chem. 1974, 17, 956-965.

[36]. Leov, B.; Snader, K. M. J. Org. Chem. 1965, 30, 1914-1916.

[37]. Jacques, J.; Eynde, V.; Delfaese, F.; Mayence, A.; Haverbeke, Y. V. Tetrahedron 1995, 51, 6511-6516.

[38]. Ghosh, S.; Saikh, F.; Das, J.; Pramanik, A. K. Tetrahedron Lett. 2013, 54, 58-62.

[39]. Furniss, B. S.; Hannaford, A. J.; Smith, P. W. G. Text book of Practical Organic Chemistry, 5thed. , Longman Singapore, 1994.

[40]. Khadilkar, B. M.; Gaikar, V. G.; Chitnavis, A. A. Tetrahedron lett. 1995, $36,8083-8086$. 